\title{
Characterization of Staphylococcus Species by Ribosomal RNA Gene Restriction Patterns
}

\author{
By MARIE-LAURE DE BUYSER,${ }^{1}+$ ANNE MORVAN, \\ FRANCINE GRIMONT ${ }^{2}$ AND NÉVINE EL SOLH ${ }^{1 *}$ \\ ${ }^{1}$ Centre National de Référence pour les Staphylocoques, Laboratoire des Staphylocoques et des \\ Streptocoques, Institut Pasteur, 75724 Paris Cedex 15, France \\ ${ }^{2}$ Unité des Entérobactéries, Institut National de la Santé et de la Recherche Médicale, Unité 199, \\ Institut Pasteur, 75724 Paris Cedex 15, France
}

(Received 17 October 1988; revised 15 December 1988; accepted 28 December 1988)

\begin{abstract}
The rRNA gene restriction patterns of 110 strains belonging to 12 staphylococcal species have been determined. The strains, isolated from various sources, were epidemiologically unrelated. Total DNA was cleaved with restriction enzymes HindIII and EcoRI, electrophoretically separated and probed with radiolabelled 16S rDNA from Bacillus subtilis inserted in a plasmid vector, pBR322. Fourty-four distinct HindIII patterns and 44 distinct EcoRI patterns were observed. Strains belonging to different species had different patterns. Although distinct patterns were also observed within some species, a core of common bands could be discerned within each species or subspecies. Analysis of the patterns revealed two taxa in Staphylococcus xylosus which were not evident using phenotypic characteristics. Of 18 strains which were difficult to identify using phenotypic schemes, 15 showed patterns typical of known species. The three remaining atypical strains showed unusual patterns and may belong either to a known species, not included in the study, or to a new species. Since various patterns were observed within some species (e.g. S. aureus and $S$. epidermidis), rRNA gene restriction patterns may have epidemiological, as well as taxonomic interest.
\end{abstract}

\section{INTRODUCTION}

In the last 14 years, the taxonomy of the genus Staphylococcus has undergone extensive changes and 26 species have been delineated by DNA-DNA hybridization (Kloos \& Schleifer, 1986; Freney et al., 1988). Although several schemes (Kloos \& Schleifer, 1986; Schleifer et al., 1984; Devriese et al., 1985; Hajek et al., 1986; Freney et al., 1988) have been recommended for the identification of staphylococcal species, many strains cannot be identified by conventional methods. Improved methods are needed to identify species, subspecies and biotypes of the genus Staphylococcus.

Staphylococci can cause severe infections. The three coagulase- and thermonucleaseproducing species Staphylococcus aureus, $S$. intermedius and $S$. hyicus often cause disease in previously healthy hosts. The so-called coagulase negative staphylococci (CNS), with the exception of $S$. saprophyticus, are opportunistic pathogens which cause infections in humans mainly after implantation of foreign bodies or in immunocompromised patients; $S$. saprophyticus is often involved in urinary tract infections (Kloos \& Schleifer, 1986; Eykyn, 1988). The increased incidence of nosocomial infections caused by $S$. aureus and CNS have

\footnotetext{
† Present address: Centre National d'Etudes Vétérinaires et Alimentaires, Laboratoire Central d'Hygiène Alimentaire, 43 rue de Dantzig, 75015 Paris, France.
}

Abbreviation: CNS, coagulase negative staphylococci.

0001-5196 (C) 1989 SGM 
stimulated interest in the use of epidemiological markers to fingerprint and compare clinical strains (Collins et al., 1984; Mickelsen et al., 1985; Parisi et al., 1986).

Recently, patterns of restriction fragments hybridizing with labelled rRNA from Escherichia coli have been used to fingerprint bacterial strains (Grimont \& Grimont, 1986; Irino et al., 1988). Probes which can be used to visualize rRNA gene restriction patterns are cloned rRNA genes (Yogev et al., 1988; Bercovier et al., 1986; Gottlieb \& Rudner, 1985; Saunders et al., 1988), 5'-end labelled rRNA (Grimont \& Grimont, 1986; Stull et al., 1988), or cDNA (Pitcher et al., 1987). Since rRNA sequences have been remarkably conserved during evolution, a single probe can be used to visualize patterns in taxonomically distant bacteria. These rRNA gene restriction patterns can be species-, subspecies- or type-specific depending on the degree of intra-species genomic heterogeneity (Grimont \& Grimont, 1986; Irino et al., 1988).

The purpose of this study was to evaluate the potential usefulness of the rRNA gene restriction pattern as a taxonomic tool and a molecular epidemiological marker for staphylococcal species of human origin or those species difficult to distinguish from humanassociated species.

\section{METHODS}

Bacterial strains. The 110 epidemiologically unrelated staphylococcal strains studied were isolated from human ( 52 clinical isolates), animal (54 isolates), hospital environment ( 2 isolates) and unknown sources ( 2 isolates). The origins of the strains are listed in Table 1. Twelve species are represented and the type strain of each species is included.

E. coli strains used were HVC45 (Dagert \& Ehrlich, 1979) harbouring plasmid pBR322 (Sutcliffe, 1978), and SF8 harbouring plasmid pBA2 (Iglesias et al., 1983). Plasmid pBA2 contains a $2.3 \mathrm{~kb}$ Bacillus subtilis DNA fragment encoding 16S rRNA inserted in the HindIII site of pBR322.

Species identification of staphylococci. (a) Identification by the French National Reference Centre for Staphylococci (FNRCS). The strains were Gram-positive and catalase-positive cocci. Staphylococci were differentiated from micrococci by use of the following tests (De Buyser $e t$ al., 1987): production of acid from glucose under anaerobic conditions; production of acid from glycerol under aerobic conditions; sensitivity to lysostaphin, nitrofurantoin, vibriostatic agent $\mathrm{O} / 129$ and bacitracin.

Isolates were assigned to known staphylococcal species according to identification schemes (Kloos \& Schleifer, 1986; Schleifer et al., 1984; Devriese et al., 1985; Hajek et al., 1988; Freney et al., 1988), by use of the following tests: colony diameter and pigment; sensitivity to novobiocin ( $5 \mu \mathrm{g}$ per disk); nitrate reduction; proteolytic activity on casein; production of urease, haemolysins, DNAase, heat-stable DNAase, clumping factor, free coagulase (rabbit plasma), alkaline phosphatase, acetoin, arginine dihydrolase, ornithine decarboxylase; production of acid under aerobic conditions from maltose, sucrose, D-trehalose, D-mannitol, D-cellobiose, lactose, D-fructose, D-melezitose, D-xylose, L-arabinose, D-raffinose, D-melibiose, D-mannose, D-turanose, D-ribose; production of acid from D-mannitol under anaerobic conditions. Staphylococcal species were also differentiated by use of the API Staph gallery (API System). S. cohnii subspecies 1 and 2 were differentiated by use of the Staph Ident gallery (API System). A seroinhibition test, with antibodies raised against $S$. aureus heat-stable DNAase (Staphynuclease kit, Biomérieux), was used to distinguish $S$. aureus nuclease from other staphylococcal nucleases.

(b) Identification by API Research Laboratory (APIRL). Sixty-eight strains were also identified by APIRL, using the ATB 32 Staph gallery (API System).

Plasmid isolation and labelling. Plasmids pBR322 and pBA2 were used as probes in the hybridization experiments. Plasmids were isolated and purified in a caesium chloride/ethidium bromide gradient according to Maniatis et al. (1982). They were labelled with $\left[\alpha{ }^{32} \mathrm{P}\right] \mathrm{dCTP}$ (Amersham) using the Multiprime DNA labelling system (Amersham) according to the manufacturer's instructions. The specific activity of the probes was approx. $10^{8}$ c.p.m. $(\mu \mathrm{g} \text { DNA) })^{-1}$.

Isolation and purification of staphylococcal DNA. Staphylococcal strains were grown in $25 \mathrm{ml}$ Brain Heart Infusion (Difco) for $18 \mathrm{~h}$ at $37^{\circ} \mathrm{C}$, then harvested and washed in $10 \mathrm{mM}$-Tris, pH 8.00, $1 \mathrm{~mm}$-EDTA (TE buffer) and resuspended in TE buffer. They were treated with lysostaphin (Sigma) at a final concentration of 2 units $\mathrm{ml}^{-1}$ for $30 \mathrm{~min}$ at $37^{\circ} \mathrm{C}$. Lysis was checked on samples treated with $1 \%$ (w/v) SDS. If lysis was incomplete, cells were further incubated at $37^{\circ} \mathrm{C}$ with lysostaphin at a final concentration of 12 units $\mathrm{ml}^{-1}$. Lysates were then treated with SDS and proteinase $\mathrm{K}$ (Merck) at final concentrations of $1 \%(\mathrm{w} / \mathrm{v})$ and 10 units $^{-1}$ respectively, for $30 \mathrm{~min}$ at $37^{\circ} \mathrm{C}$. Potassium acetate was added at a final concentration of $1 \mathrm{M}$ and the mixtures were incubated for $30 \mathrm{~min}$ at $4^{\circ} \mathrm{C}$. Proteins were denatured and removed by sequential extraction with equal volumes of phenol, phenol/chloroform/isoamyl alcohol (25:24:1, by vol.) and chloroform/isoamyl alcohol (24:1, v/v). 2-Propanol ( 0.6 vol.) was added to the aqueous layer. Precipitated DNA was recovered by centrifugation, washed in $70 \%(\mathrm{v} / \mathrm{v})$ ethanol, dried and resuspended in TE buffer, treated with RNAase (Calbiochem, Behring Diagnostics) at a final concentration of 3 units $\mathrm{ml}^{-1}$ for $2 \mathrm{~h}$ at $37^{\circ} \mathrm{C}$, and kept at $-20^{\circ} \mathrm{C}$ until use. 
Table 1. Origin and $r R N A$ gene restriction patterns of 110 staphylococcal strains

\begin{tabular}{|c|c|c|c|c|}
\hline $\begin{array}{c}\text { Taxon and strain } \\
\text { designation }\end{array}$ & $\begin{array}{l}\text { Origin* or } \\
\text { reference }\end{array}$ & Source & $\begin{array}{c}\text { HindIII rRNA } \\
\text { gene restriction } \\
\text { pattern }\end{array}$ & $\begin{array}{r}E c o R I r R \\
\text { gene restric } \\
\text { pattern }\end{array}$ \\
\hline \multicolumn{5}{|l|}{ S. aureus } \\
\hline ATCC $12600 \dagger$ & ATCC & Man & $\mathrm{H}_{1}$ & $E_{1}$ \\
\hline RN 450 & Novick (1967) & Unknown & $\mathrm{H}_{1}$ & $\mathrm{E}_{1}$ \\
\hline BM $3250 \ddagger$ & El Solh et al. (1984) & Man & $\mathrm{H}_{1}$ & $\mathrm{E}_{1}$ \\
\hline BM $3254 \ddagger$ & El Solh et al. (1984) & Man & $\mathrm{H}_{1}$ & $E_{1}$ \\
\hline BM $3121 \ddagger$ & El Solh et al. (1981) & Man & $\mathrm{H}_{1}$ & $\mathrm{E}_{1}$ \\
\hline ATCC 6538P & ATCC & Unknown & $\mathrm{H}_{1}$ & $\mathrm{E}_{2}$ \\
\hline BM $3247 \ddagger$ & El Solh et al. (1984) & Man & $\mathrm{H}_{2}$ & $\mathrm{E}_{3}$ \\
\hline $\mathrm{CH} 1$ & De Buyser et al. (1987) & Goat & $\mathbf{H}_{3}$ & $\mathrm{E}_{4}$ \\
\hline CH 96 & De Buyser et al. (1987) & Goat & $\mathbf{H}_{3}$ & $\mathrm{E}_{4}$ \\
\hline CH 102 & De Buyser et al. (1987) & Goat & $\mathbf{H}_{3}$ & $\mathrm{E}_{4}$ \\
\hline CH 54 & De Buyser et al. (1987) & Goat & $\mathbf{H}_{3}$ & $E_{4}$ \\
\hline $\mathrm{CH} 31$ & De Buyser et al. (1987) & Goat & $\mathrm{H}_{4}$ & $\mathbf{E}_{4}$ \\
\hline $\mathrm{CH} 73$ & De Buyser et al. (1987) & Goat & $\mathrm{H}_{4}$ & $\mathrm{E}_{4}$ \\
\hline $\mathrm{CH} 49$ & De Buyser et al. (1987) & Goat & $\mathbf{H}_{5}$ & $\mathrm{E}_{4}$ \\
\hline $\mathrm{CH} 74$ & De Buyser et al. (1987) & Goat & $\mathrm{H}_{6}$ & $\mathrm{E}_{4}$ \\
\hline 64048 & Pillet \& Orta (1973) & Poultry & $\mathrm{H}_{7}$ & $\mathrm{E}_{5}$ \\
\hline 64043 & Pillet \& Orta (1973) & Poultry & $\mathrm{H}_{7}$ & $\mathrm{E}_{6}$ \\
\hline BM 3319 & El Solh et al. (1984) & Man & $\mathrm{H}_{7}$ & $\mathrm{E}_{7}$ \\
\hline 86315 & FNRCS & Man & $\mathbf{H}_{7}$ & $E_{8}$ \\
\hline BM 3251 & El Solh et al. (1984) & Man & $\mathrm{H}_{7}$ & $\mathrm{E}_{8}$ \\
\hline 86002 & FNRCS & Man & $\mathrm{H}_{7}$ & $\mathbf{E}_{9}$ \\
\hline BM 3248 & El Solh et al. (1984) & Man & $\mathrm{H}_{8}$ & $E_{10}$ \\
\hline 85282 & FNRCS & Man & $\mathrm{H}_{8}$ & $E_{10}$ \\
\hline 85522 & FNRCS & Man & $\mathrm{H}_{8}$ & $E_{11}$ \\
\hline BM $3152 \ddagger$ & El Solh et al. (1981) & Man & $\mathrm{H}_{9}$ & $E_{12}$ \\
\hline \multicolumn{5}{|l|}{$\begin{array}{l}\text { S. intermedius } \\
\text { subsp. pigeon }\end{array}$} \\
\hline $\begin{array}{l}\text { ATCC } 29663+ \\
\text { subsp. carnivora }\end{array}$ & ATCC & Pigeon & $\mathbf{H}_{10}$ & $\mathrm{E}_{13}$ \\
\hline 81508 & FNRCS & Dog & $\mathbf{H}_{11}$ & $E_{14}$ \\
\hline 61221 & I. Live & Dog & $\mathrm{H}_{12}$ & $\mathrm{E}_{15}$ \\
\hline 74337 & FNRCS & Dog & $\mathrm{H}_{12}$ & $\mathrm{E}_{15}$ \\
\hline 75619 & FNRCS & Dog & $\mathbf{H}_{12}$ & $\mathrm{E}_{15}$ \\
\hline 83743 & FNRCS & Man & $\mathrm{H}_{12}$ & $E_{15}$ \\
\hline \multicolumn{5}{|l|}{ S. hyicus } \\
\hline ATCC $11249 \dagger$ & L. A. Devriese & Pig & $\mathbf{H}_{13}$ & $E_{16}$ \\
\hline VA 503 & L. A. Devriese & Pig & $\mathrm{H}_{14}$ & $E_{16}$ \\
\hline BOD 28 & L. A. Devriese & Cow & $\mathrm{H}_{14}$ & $E_{16}$ \\
\hline E 80 & FNRCS & Cow & $\mathbf{H}_{15}$ & $E_{17}$ \\
\hline PA 81 & L. A. Devriese & Horse & $\mathrm{H}_{16}$ & $E_{18}$ \\
\hline VII 96 & L. A. Devriese & Poultry & $\mathrm{H}_{17}$ & $\mathrm{E}_{19}$ \\
\hline \multicolumn{5}{|l|}{ S. chromogenes } \\
\hline NCTC $10530 \dagger$ & L. A. Devriese & Pig & $\mathbf{H}_{18}$ & $\mathbf{E}_{20}$ \\
\hline VA 608 & L. A. Devriese & Pig & $\mathbf{H}_{19}$ & $\mathrm{E}_{20}$ \\
\hline BO 64 & L. A. Devriese & Cow & $\mathbf{H}_{19}$ & $\mathbf{E}_{20}$ \\
\hline BO 153 & L. A. Devriese & Cow & $\mathbf{H}_{19}$ & $\mathrm{E}_{20}$ \\
\hline $\mathrm{CH} 22$ & De Buyser et al. (1987) & Goat & $\mathbf{H}_{19}$ & $\mathrm{E}_{20}$ \\
\hline $\mathrm{CH} 62$ & De Buyser et al. (1987) & Goat & $\mathrm{H}_{19}$ & $\mathrm{E}_{20}$ \\
\hline \multicolumn{5}{|l|}{ S. epidermidis } \\
\hline ATCC $14990 \dagger$ & W. E. Kloos & Man & $\mathbf{H}_{20}$ & $\mathrm{E}_{21}$ \\
\hline 85711 & FNRCS & Man & $\mathbf{H}_{20}$ & $E_{21}$ \\
\hline 86166 & FNRCS & Man & $\mathrm{H}_{20}$ & $E_{21}$ \\
\hline 86060 & FNRCS & Man & $\mathrm{H}_{21}$ & $E_{21}$ \\
\hline 52260 & Pillet \& Orta (1970) & Man & $\mathrm{H}_{22}$ & $E_{21}$ \\
\hline 75864 & FNRCS & Man & $\mathrm{H}_{22}$ & $\mathrm{E}_{21}$ \\
\hline 84331 & FNRCS & Man & $\mathrm{H}_{22}$ & $\mathbf{E}_{21}$ \\
\hline $\mathrm{CH} 8$ & De Buyser et al. (1987) & Goat & $\mathrm{H}_{22}$ & $\mathrm{E}_{21}$ \\
\hline $\mathrm{CH} 93$ & De Buyser et al. (1987) & Goat & $\mathrm{H}_{22}$ & $E_{21}$ \\
\hline CH 106 & De Buyser et al. (1987) & Goat & $\mathrm{H}_{22}$ & $E_{21}$ \\
\hline
\end{tabular}


Table 1. (continued)

Taxon and strain designation

S. epidermidis - contd

CH 109

CH 133

CH 145

CH 58

S. warneri

ATCC 27836†

MCS 4

75594

76066

CH 251

$\mathrm{CH} 108$

CH 98

CH 225

CH 17

CH 30

CH 60

Unidentified $\$$

CH 199

83796

S. hominis 1\|

ATCC 27844†
SM 67
75611
78354
86116

S. hominis 2\|

CH 181

S. lugdunensis

ATCC $43809 \dagger$

CRS 307

CH 97

CRSN 850412

83091

86428

CH 80

84373

$S$. haemolyticus

ATCC $29970+$

KES 23

75581

77028

85474

85333

S. saprophyticus ATCC $15305 \dagger$

KL 20

75168

75751

S. xylosus

ATCC $29971 \dagger$
CH 167
CH 61
CH 232
77020
78037
CH 65
CH 100

Origin* or reference

Source

De Buyser et al. (1987)

De Buyser et al. (1987)

De Buyser et al. (1987)

De Buyser et al. (1987)

W. E. Kloos

W. E. Kloos

FNRCS

FNRCS

De Buyser et al. (1987)

De Buyser et al. (1987)

De Buyser et al. (1987)

De Buyser et al. (1987)

De Buyser et al. (1987)

De Buyser et al. (1987)

De Buyser et al. (1987)

De Buyser et al. (1987)

FNRCS

W. E. Kloos

W. E. Kloos

FNRCS

FNRCS

FNRCS

De Buyser et al. (1987)

J. Freney

J. Freney

De Buyser et al. (1987)

J. Freney

FNRCS

FNRCS

De Buyser et al. (1987)

FNRCS

Goat

Goat

Goat

Goat

Man

Man

Man

Man

Goat

Goat

Goat

Goat

Goat

Goat

Goat

Goat

Man

Man

Man

Man

Man

Man

Goat

Man

Man

Goat

Man

Man

Man

Goat

Man

W. E. Kloos

W. E. Kloos

FNRCS

FNRCS

FNRCS

FNRCS

W. E. Kloos

W. E. Kloos

FNRCS

FNRCS

W. E. Kloos

De Buyser et al. (1987)

De Buyser et al. (1987)

De Buyser et al. (1987)

FNRCS

FNRCS

De Buyser et al. (1987)

De Buyser et al. (1987)

Man

Man

Man

Man

Man

Man

Man

Man

Man

Man

Man

Goat

Goat

Goat

Horse

Horse

Goat

Goat
HindIII rRNA

gene restriction pattern

EcoRI rRNA

gene restriction pattern

$E_{21}$

$\mathrm{H}_{22}$

$\begin{array}{ll}\mathrm{H}_{22} & \mathrm{E}_{21} \\ \mathrm{H}_{23} & \mathrm{E}_{21}\end{array}$

$\mathrm{H}_{24} \quad \mathrm{E}_{22}$

$\mathrm{H}_{24} \quad \mathrm{E}_{22}$

$\mathrm{H}_{24} \quad \mathrm{E}_{22}$

$\mathrm{H}_{24}$

$\mathrm{H}_{24} \quad \mathrm{E}_{22}$

$\mathrm{H}_{24} \quad \mathrm{E}_{22}$

$\mathrm{H}_{24} \quad \mathrm{E}_{22}$

$\mathrm{H}_{24} \quad \mathrm{E}_{22}$

$\mathrm{H}_{25} \quad \mathrm{E}_{22}$

$\mathrm{H}_{25} \quad \mathrm{E}_{22}$

$\mathrm{H}_{25} \quad \mathrm{E}_{22}$

$\mathrm{H}_{26} \quad \mathrm{E}_{23}$

$\mathrm{H}_{26} \quad \mathrm{E}_{23}$

$\mathrm{H}_{27} \quad \mathrm{E}_{24}$

$\mathrm{H}_{27} \quad \mathrm{E}_{24}$

$\mathrm{H}_{27} \quad \mathrm{E}_{24}$

$\mathrm{H}_{27} \quad \mathrm{E}_{24}$

$\mathrm{H}_{28} \quad \mathrm{E}_{25}$

$\mathrm{H}_{29} \quad \mathrm{E}_{26}$

$\mathrm{H}_{30} \quad \mathrm{E}_{27}$

$\mathrm{H}_{30} \quad \mathrm{E}_{27}$

$\mathrm{H}_{31} \quad \mathrm{E}_{27}$

$\mathrm{H}_{31} \quad \mathrm{E}_{27}$

$\mathrm{H}_{31} \quad \mathrm{E}_{27}$

$\mathrm{H}_{31} \quad \mathrm{E}_{27}$

$\mathrm{H}_{31} \quad \mathrm{E}_{28}$

$\mathrm{H}_{32}$

$\mathrm{H}_{32}$

$\mathrm{H}_{32}$

$\mathrm{H}_{32}$

$\mathrm{H}_{33}$

$\mathrm{H}_{34}$

$\mathrm{E}_{29}$

$\mathrm{E}_{29}$

$\mathrm{E}_{29}$

$\mathrm{E}_{29}$

$\mathrm{E}_{29}$

$\mathbf{E}_{30}$

$\mathrm{H}_{35}$

$\mathrm{H}_{35}$

$\mathrm{H}_{35}$

$E_{31}$

$\mathrm{E}_{31}$

$E_{31}$
$E_{31}$
$\mathrm{H}_{36} \quad \mathrm{E}_{32}$

$\begin{array}{ll}\mathrm{H}_{37} & \mathrm{E}_{33}\end{array}$

$\mathrm{H}_{37} \quad \mathrm{E}_{33}$

$\mathrm{H}_{37} \quad \mathrm{E}_{33}$

$\mathrm{H}_{38} \quad \mathrm{E}_{34}$

$\mathrm{H}_{38} \quad \mathrm{E}_{35}$ 
Table 1. (continued)

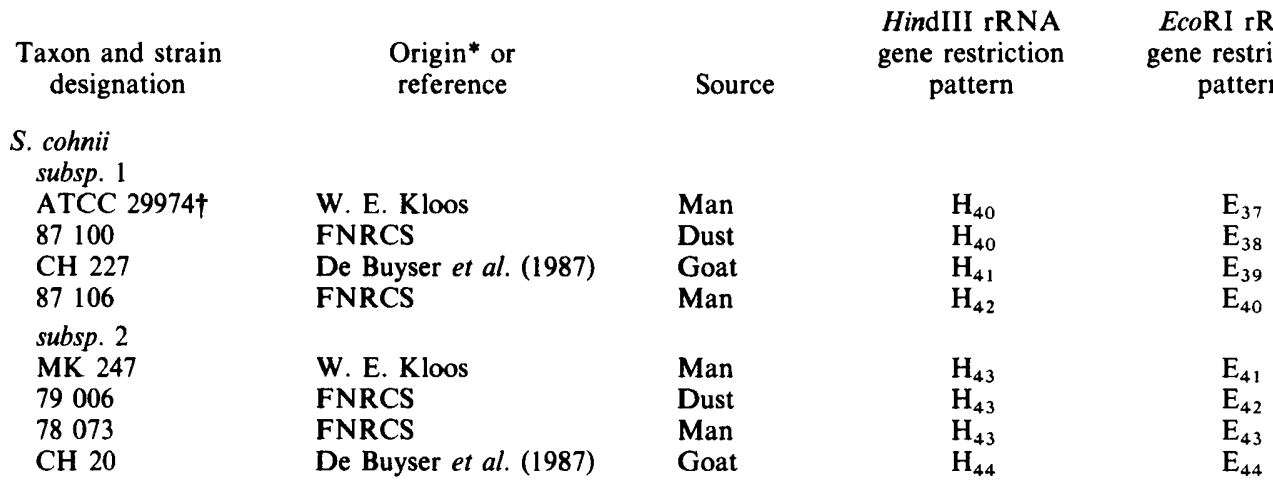

* ATCC, American Type Culture Collection, Rockville, Md, USA; NCTC, National Collection of Type Cultures, London UK; FNRCS, National Reference Centre for Staphylococci, Paris, France; I. Live, Philadelphia, Pa., USA; L. A. Devriese, Gent, Belgium; W. E. Kloos, Raleigh, NC, USA; J. Freney, Lyon, France.

† Type strain.

$\ddagger$ Methicillin-resistant $S$. aureus strains isolated in French hospitals. All the other $S$. aureus strains studied were methicillin-susceptible.

$\S$ The two unidentified strains could correspond either to $S$. warneri or to $S$. hominis according to their biochemical characteristics. Their rRNA gene restriction patterns were intermediate between those observed with $S$. warneri and with $S$. hominis.

$\| S$. hominis 1 and $S$. hominis 2 were differentiated according to the ATB 32 Staph gallery (API System) by use of three biochemical characteristics: production of alkaline phosphatase $(1 \%$ of $S$. hominis 1 strains and $94 \%$ of $S$. hominis 2 strains are positive), production of arginine dihydrolase $(5 \%$ of $S$. hominis 1 strains and $73 \%$ of $S$. hominis 2 strains are positive), and production of acid from mannose ( $1 \%$ of $S$. hominis 1 strains and $75 \%$ of $S$. hominis 2 strains are positive).

Restriction endonuclease digestion and agarose gel electrophoresis of DNA. DNA (2 to $5 \mu \mathrm{g}$ ) was cleaved with restriction endonuclease HindIII (Amersham) or EcoRI (Genofit), according to the manufacturer's instructions. Restriction DNA fragments were separated electrophoretically in $0.8 \%(\mathrm{w} / \mathrm{v})$ agarose (Sigma) gel in Tris/borate buffer as described by Maniatis et al. (1982).

$B g l \mathrm{II}$ and HindIII-EcoRI fragments of bacteriophage $\lambda$ DNA (Biolabs) were used as size markers. The sizes of these fragments were the same as those published by Maniatis et al. (1982).

Southern blotting and hybridization. After electrophoresis, agarose gels were sequentially soaked in $0.25 \mathrm{M}-\mathrm{HCl}$ for $5 \mathrm{~min}, 1.5 \mathrm{M}-\mathrm{NaCl}, 0.5 \mathrm{M}-\mathrm{NaOH}$ for $30 \mathrm{~min}$ and $0.5 \mathrm{M}-\mathrm{Tris} / \mathrm{HCl}$ (pH 7.2), 1.5 M- NaCl, $1 \mathrm{~mm}-\mathrm{EDTA}$ for $30 \mathrm{~min}$. Southern blotting (Southern, 1975) was carried out on Hybond-N nylon membrane (Amersham). The blotted DNA was fixed by UV irradiation at $305 \mathrm{~nm}$ for $5 \mathrm{~min}$. The prehybridization, hybridization and washing steps were performed as previously described (Grimont \& Grimont, 1986). The blots were exposed to Fuji RX films at $-70^{\circ} \mathrm{C}$ with intensifying screens for $24 \mathrm{~h}$ to several days.

\section{RESULTS}

\section{Biochemical identification of staphylococcal species}

Identification showed discrepancies in $12(17.6 \%)$ out of 68 strains identified at both FNRCS and APIRL. Strain 64048 identified with S. aureus (FNRCS) or S. chromogenes (APIRL). This strain showed delayed production of free coagulase and was clumping-factor-positive, acetoinnegative and heat-stable-DNAase-positive. Activity of this latter enzyme was inhibited by antiserum raised against $S$. aureus heat-stable DNAase. Strains $\mathrm{CH} 133$ and $\mathrm{CH} 145$, which showed delayed aerobic acid production from sucrose, identified with $S$. epidermidis (FNRCS) or was unidentified (APIRL). Three strains (CH98, CH225 and 83796) identified with $S$. hominis (FNRCS) or $S$. warneri (APIRL), whereas strain 85474 identified with $S$. hominis (FNRCS) or S. haemolyticus (APIRL). Five strains (CH108, CH17, CH60, CH199 and CH181) 


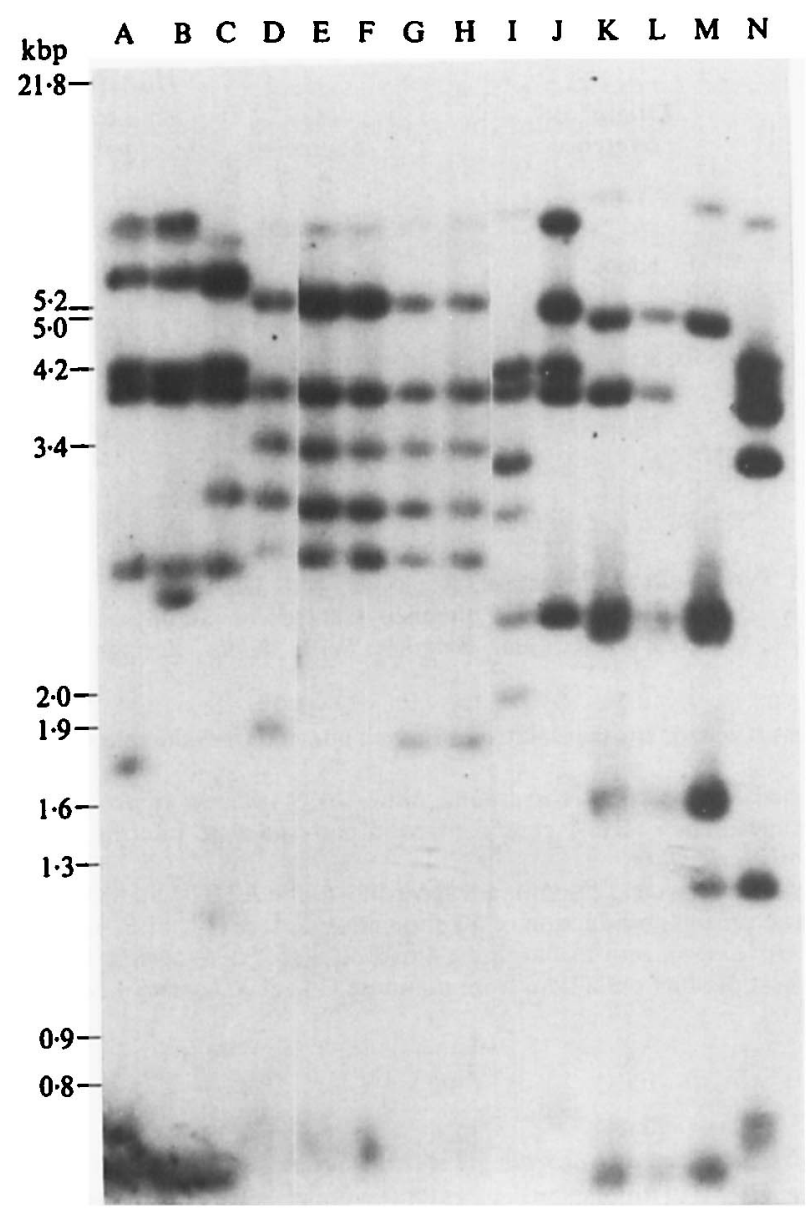

Fig. 1. Autoradiogram of HindIII digests of DNA from 14 staphylococcal strains, separated by electrophoresis and probed with ${ }^{32} \mathrm{P}$-labelled plasmid pBA2. Lanes A to C, S. haemolyticus strains ATCC 29970, 85474, 85333; lanes D to H, S. warneri strains CH251, CH30, CH60, CH108, CH98; Lane I, S. hominis 1 strain ATCC 27844; lane J, S. hominis 2 strain $\mathrm{CH} 181$; lanes K to $\mathrm{M}, S$. lugdunensis strains 86428, CH80, CH97; lane N, unidentified strain $\mathrm{CH} 199$.

identified with either $S$. warneri or $S$. hominis without possible distinction between the species (FNRCS); however, they identified with either $S$. warneri (CH108, CH17, CH60, CH199) or $S$. hominis $2(\mathrm{CH} 181)$ at APIRL.

Although identified without discrepancy at both FNCRS and APIRL, three $S$. aureus strains and three $S$. lugdunensis strains were found to be atypical. The three $S$. aureus strains were atypical with respect to one or two characteristics that are usually shared by more than $90 \%$ of the $S$. aureus strains (Kloos \& Schleifer, 1986). Strain 64043 produced no heat-stable DNAase. Strain $\mathrm{CH} 96$ failed to produce acid from mannitol under anaerobic conditions. Strain 86315 was acetoin-negative and free coagulase-negative. Heat-stable DNAase was produced by the latter two atypical strains. Enzyme activity was inhibited by antiserum raised against $S$. aureus heatstable DNAase, which confirmed that these two strains belonged to $S$. aureus species. The three $S$. lugdunensis strains (CH97, 86428 and $\mathrm{CH} 80)$ were clumping-factor-negative even when tested on the recommended medium, Columbia blood agar (Freney et al., 1988). 


\section{$r R N A$ gene restriction patterns}

When the HindIII- or EcoRI-cleaved staphylococcal DNA was probed with pBR322, no restriction fragments hybridized with the probe. Hence, pBA2 was used as a probe for the visualization of rRNA genes without the need for separating the insert from the pBR 322 vector.

The patterns of radiolabelled bands hybridizing with $\mathrm{pBA} 2$ were designated the rRNA gene restriction patterns. HindIII rRNA gene restriction patterns of 13 strains belonging to four species and one unidentified strain are shown in Fig. 1. A total of 44 distinct HindIII rRNA gene restriction patterns, designated $\mathrm{H} 1$ to $\mathrm{H} 44$, and 44 distinct EcoRI rRNA gene restriction patterns, designated E1 to E44, could be distinguished (Table 1). As shown in Figs 2 and 3, HindIII patterns consisted of 5 to 10 bands whereas EcoRI patterns consisted of 6 to 13 bands. Each species showed 1 to 9 HindIII patterns and 1 to 12 EcoRI patterns. Strains belonging to different species did not share the same pattern.

Among $S$. aureus strains, the goat isolates belonging to biotype ovis, $\mathrm{CH} 1, \mathrm{CH} 96, \mathrm{CH} 102$, CH54, CH31, CH73, CH49 and CH74 (De Buyser et al., 1987), specifically showed a single EcoRI pattern, E4, and four HindIII patterns, H3, H4, H5 and H6, which were not shown by the other $S$. aureus strains. The five methicillin-resistant $S$. aureus strains studied showed three distinct pairs of HindIII- and EcoRI-patterns, H1-E1, H2-E3 and H9-E12. One of these pairs of patterns, H1-El, was also shown by methicillin-susceptible $S$. aureus strains (Table 1).

Each species could be characterized by a core of common rRNA gene restriction fragments which constituted a species-specific pattern. However, when $S$. cohnii DNA was cleaved by HindIII, no species-specific pattern was observed but subspecies-specific patterns could be detected. Strains of $S$. cohnii subspecies 1 (Kloos \& Schleifer, 1986) shared two HindIII fragments and this pair of fragments was not found in other patterns. Strains of $S$. cohnii subspecies 2 showed six common HindIII fragments. When considering the eight $S$. xylosus strains studied, only one and three common bands were shown among HindIII- and EcoRIpatterns, respectively. However, on the basis of their HindIII- and EcoRI-patterns, two taxa could be distinguished among these strains. The first taxon included five strains showing patterns H36, H37 (five common bands) and E32, E33 (eight common bands). The second one included three strains showing patterns H38, H39 (six common bands) and E34, E35, E36 (nine common bands). When considering $S$. intermedius strains, only two common bands were observed among EcoRI patterns, whereas eight common bands were observed within $S$. intermedius subsp. carnivora EcoRI patterns.

The characterization of taxon-specific cores of fragments among rRNA gene restriction patterns was found useful in assessing the identification of the six atypical strains and to identify nine out of the 12 strains for which discrepant results were obtained. According to rRNA gene restriction patterns, strains 64048, 64043, CH96 and 86315 identified with $S$. aureus; $\mathrm{CH} 133$ and $\mathrm{CH} 145$ with S. epidermidis; $\mathrm{CH} 108, \mathrm{CH} 98, \mathrm{CH} 225, \mathrm{CH} 17$ and $\mathrm{CH} 60$ with $S$. warneri; CH97, 86428 and CH80 with S. lugdunensis, and 85474 with S. haemolyticus. Strains CH199 and 83796, which were found biochemically to be either $S$. warneri or $S$. hominis, showed intermediate patterns (H26, E23) between those of these two species and could not be identified. Strain $\mathrm{CH} 181$, the only strain which identified with $S$. hominis 2 (a group detected using ATB 32 Staph gallery but not yet validated by molecular studies), showed different patterns (H28, E25) from that shown by the five strains identified with $S$. hominis 1 and the 11 identified with $S$. warneri.

\section{DISCUSSION}

The discrepancies in identification obtained at FNRCS and APIRL concerned $17.6 \%$ of the 68 staphylococcal strains studied in both laboratories. This indicates the insufficiency of present identification schemes based on phenotypic characteristics.

rRNA gene restriction patterns have been proposed as a taxonomic aid mainly for Gramnegative bacteria (Grimont \& Grimont, 1986; Saunders et al., 1988), and therefore it was interesting to evaluate the contribution of these patterns to the identification of Gram-positive bacteria such as staphylococci. Using this method, species- or subspecies-specific cores of restriction fragments have been observed. Thus, species usually considered as difficult to 


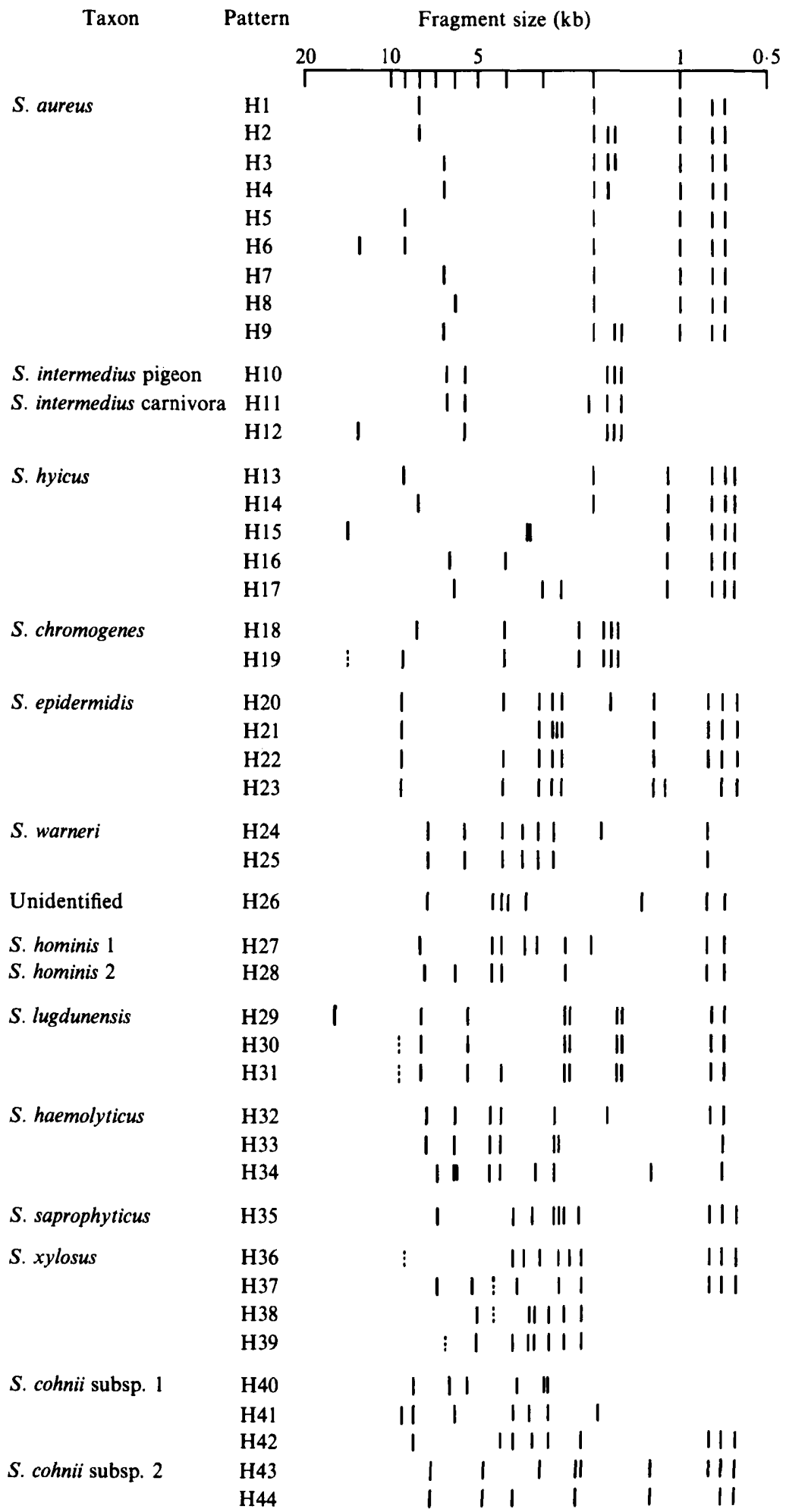

Fig. 2. Schematic representation of the 44 HindIII rRNA gene restriction patterns ( $\mathrm{H} 1$ to H44) detected among the 110 staphylococcal strains studied. Dotted lines indicate very weakly labelled bands. 
Taxon

S. aureus

$S$. intermedius pigeon

$S$. intermedius carnivora

E14

E15

S. hyicus

S. chromogenes

$S$. epidermidis

S. warneri

Unidentified

S. hominis 1

S. hominis 2

S. lugdunensis

S. haemolyticus

S. saprophyticus

S. xylosus

S. cohnii subsp. 2

Pattern

E20

E21

E22

E23

E25

E26

E2

E28

Fragment size (kb)

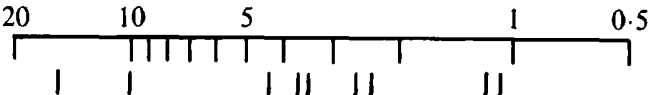

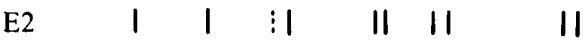

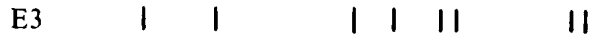

$\begin{array}{llllllllllllll}\text { E4 } & \text { I } & 1 & 1 & \text { II } & \text { II }\end{array}$

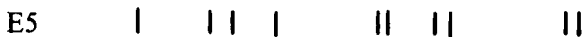

$\begin{array}{lllllllllllllll} & \text { E6 } & 1 & 1 & 1 & 1 & 11 & \text { II }\end{array}$

E7 I 11 li $11 \quad$ I 11

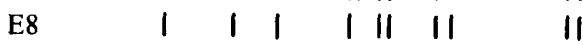

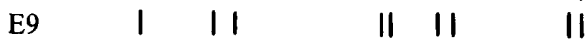

$\begin{array}{lllllll}\text { E10 I I I I } & \text { I } & \text { II } & \text { II }\end{array}$

$\begin{array}{lllllll}\text { E11 } & \text { I } & \text { I } & \text { I } & \| & 1 & \text { II }\end{array}$

$\begin{array}{llllllll}E 12 & \mid & \mid & \mid & \| & \| 1 & \mid\end{array}$

$\begin{array}{lllllll} & 13 & 1 & 1 & 1 & 11\end{array}$

E16 I II III : I

E17 I I || I||

$\begin{array}{lllllll}\text { E18 } & \text { I } & \text { I } & \text { II } & \text { II } & \text { I } & \text { I }\end{array}$

$\begin{array}{llllll} & 19 & \text { I } & \text { II } & \text { I }\end{array}$

E24 I

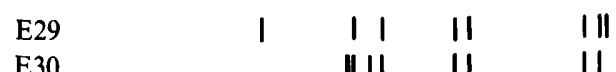

| I | | |||

$$
11
$$$$
\text { I I I III }
$$

III

II I

$\begin{array}{llllll}1 & 1 & 1 & 1 & \text { || } & \text { II }\end{array}$

$\begin{array}{lllllll}1 & 1 & 1 & 11 & 11\end{array}$

6

$\begin{array}{lll}1 & 1 & 1 \\ 1 & 1 & 1 \\ 1 & 1 & 1\end{array}$

III III III

$\begin{array}{lll}11 & 111 & 111\end{array}$

E30 III II

E31 1 I 11111 III

E32 II I III III

E33 I II 1 II III

E34 I I II II I III

E35 I I I II II I III

E36 I I I II II III

\begin{tabular}{|c|c|c|c|c|c|c|c|}
\hline \multirow[t]{4}{*}{ S. cohnii subsp. 1} & E37 & 1 & II & II & II & III & III \\
\hline & E38 & I & II & & 11 & II & III \\
\hline & E39 & 1 & 1 & & II & 11 & III \\
\hline & $\mathrm{E} 40$ & 11 & & 1 & 11 & 11 & III \\
\hline \multirow[t]{4}{*}{ S. cohnii subsp. 2} & E41 & 1 & 1 & & II & 11 & III \\
\hline & $\mathrm{E} 42$ & 1 & & & 11 & 11 & 111 \\
\hline & $\mathrm{E} 43$ & 1 & 1 & & II & 11 & III \\
\hline & E44 & 1 & I & & II & 1 & III \\
\hline
\end{tabular}

Fig. 3. Schematic representation of the 44 EcoRI rRNA gene restriction patterns (E1 to E44) detected among the 110 staphylococcal strains studied. Dotted lines indicate very weakly labelled bands. 
distinguish from each other using phenotypic characteristics, $S$. aureus, S. intermedius, S. hyicus and $S$. chromogenes on the one hand, $S$. haemolyticus, $S$. warneri and $S$. hominis on the other, could be easily differentiated. Moreover, two distinct taxa could be discerned among the $S$. xylosus strains studied on the basis of their rRNA gene restriction patterns, whereas the phenotypic charcteristics did not enable differentiation of these taxa. S. xylosus strains should therefore be submitted to quantitative DNA-DNA hybridization in order to assess the validity of these two taxa. In addition, the use of rRNA gene restriction patterns enabled the identification of 15 out of 18 phenotypically atypical strains. The three remaining phenotypically atypical strains (CH199, 83796 and $\mathrm{CH} 181)$ had unusual patterns and could belong either to a species not included in this study or to a new species or subspecies. They should also be submitted to quantitative DNA-DNA hybridization for further elucidation of their taxonomic position.

The present work concerned staphylococcal species frequently involved in human and animal infection and other species from which they need to be differentiated. rRNA gene restriction patterns of remaining staphylococcal species are now under study in our laboratory.

Genomic species (i.e. species defined by DNA relatedness) may show various degrees of heterogeneity. Homogeneous species often show one or a few rRNA gene restriction patterns (Grimont \& Grimont, 1986) whereas heterogeneous species show many patterns (Irino et al., 1988). Thus, in addition to taxonomic relevance, rRNA gene restriction patterns may have epidemiological relevance (Grimont \& Grimont, 1986; Stull et al., 1988; Irino et al., 1988). In this study, a variety of patterns was observed within some species, depending on the restriction enzyme used to cleave DNA. Cleavage with EcoRI yielded 12 distinct patterns among the $25 S$. aureus strains studied whereas only nine HindIII patterns were observed. In contrast, among the $14 S$. epidermidis strains, the number of patterns was greater when HindIII was used (four distinct patterns) instead of EcoRI (single pattern). The variety of patterns observed among $S$. aureus strains, especially the methicillin-resistant ones, and the $S$. epidermidis strains, could be used to type these strains. However, such an epidemiological approach needs to be validated by study of many strains involved in different outbreaks.

In conclusion, the present work demonstrates the usefulness of rRNA gene restriction patterns in staphylococcal taxonomy. They may be used as a screening method to detect new taxa. The resulting banding patterns may also constitute additional epidemiological markers.

We thank P. A. D. Grimont for stimulating comments and O. Rouelland for secretarial assistance.

\section{REFERENCES}

Bercovier, H., Kafri, O. \& Sela, S. (1986). Mycobacteria possess a surprisingly small number of ribosomal RNA genes in relation to the size of their genome. Biochemical and Biophysical Research Communications 136, 1136-1141.

Collins, J. K., SMith, J. S. \& Kelly, M. T. (1984). Comparison of phage typing, plasmid mapping, and antibiotic resistance patterns as epidemiologic markers in a nosocomial outbreak of methicillinresistant Staphylococcus aureus infections. Diagnostic Microbiology of Infectious Diseases 2, 233245.

Dagert, M. \& Ehrlich, S. D. (1979). Prolonged incubation in calcium chloride improves the competence of Escherichia coli cells. Gene 6, 23-28.

De Buyser, M. L., Dilasser, F., Hummel, R. \& BERGDOLL, M. S. (1987). Enterotoxin and toxic shock syndrome toxin-1 production by staphylococci isolated from goat's milk. International Journal of Food Microbiology 5, 301-309.

Devriese, L. A., Schleifer, K. H., \& Adegoke, G. O. (1985). Identification of coagulase-negative staphy- lococci from farm animals. Journal of Applied Bacteriology 58, 45-55.

El Solh, N., Fouace, J. M., Pillet, J. \& Chabbert, Y. A. (1981). Plasmid DNA content of multiresistant Staphylococcus aureus strains. Annales de microbiologie 132B, 131-156.

El Solh, N., Bismuth, R., Allignet, J. \& Fouace, J. M. (1984). Résistance à la pristinamycine (ou virginiamycine) des souches de Staphylococcus aureus. Pathologie et Biologie 32, 362-368.

EYXYN, S. J. (1988). Staphylococcal sepsis. The changing pattern of disease and therapy. Lancet $\mathbf{i}$, 100-104

Freney, J., Brun, Y., Bes, M., Meugnier, H., Grimont, F., Grimont, P. A. D., Nervi, C. \& FleuretTe, J. (1988). Staphylococcus lugdunensis sp. nov. and Staphylococcus schleiferi sp. nov., two species from human clinical specimens. International Journal of Systematic Bacteriology 38, 168172.

Gottlieb, P. \& Rudiser, R. (1985). Restriction site polymorphism of ribosomal ribonucleic acid gene 
sets in members of the Genus Bacillus. International Journal of Systematic Bacteriology 35, 244-252.

Grimont, F. \& GRIMONT, P. A. D. (1986). Ribosomal ribonucleic acid gene restriction patterns as potential taxonomic tools. Annales de l'Institut Pasteur/ Microbiologie 137B, 165-175.

Hajex, V., Devriese, L. A., Mordarski, M., Goodfellow, M., Pulverer, G. \& Varaldo, P. E. (1986). Elevation of Staphylococcus hyicus subsp. chromogenes (Devriese et al., 1978) to species status: Staphylococcus chromogenes (Devriese et al., 1978) comb. nov. Systematic and Applied Microbiology 8, 169-173.

Iglesias, A., Ceglowski, P. \& Trautner, T. A. (1983). Plasmid transformation in Bacillus subtilis. Effects of the insertion of Bacillus subtilis rRNA genes into plasmids. Molecular and General Genetics 192, 149-155.

irino, K., Grimont, F., Casin, I., Grimont, P. A. D. \& The Brazilian Purpuric Fever Study Group (1988). rRNA gene restriction patterns of Haemophilus influenzae biogroup aegyptius strains associated with Brazilian purpuric fever. Journal of Clinical Microbiology 26, 1535-1538.

Kloos, W. E. \& Schleifer, K. H. (1986). Genus IV. Staphylococcus Rosenbach 1884, $18^{\mathrm{AL}}$, (Nom. Cons. Opin. 17 Jud. Comm. 1958. 153). In: Bergey's Manual of Systematic Bacteriology, vol, 2, pp. 10131035. Edited by P. H. A. Sneath. Baltimore: Williams \& Wilkins.

Maniatis, T., Fritsch, E. F. \& Sambrook, J. (1982). Molecular Cloning: a Laboratory Manual. Cold Spring Harbor, NY: Cold Spring Harbor Laboratory.

Mickelsen, P. A., Plorde, J. J., Gordon, K. P., Hargiss, C., MCClure, J., SCHOENKNECht, F. D., Condie, F., Tenover, F. C. \& Tompkins, L. S. (1985). Instability of antibiotic resistance in a strain of Staphylococcus epidermidis isolated from an outbreak of prosthetic valve endocarditis. Journal of Infectious Diseases 152, 50-57.

Novick, R. P. (1967). Properties of a cryptic high frequency transducing phage in $S$. aureus. Virology 33, $155-166$.
Parisi, J. T., Lampson, B. C., HoOver, D. L. \& Khan, J. A. (1986). Comparison of epidemiologic markers for Staphylococcus epidermidis. Journal of Clinical Microbiology 24, 56-60.

Pillet, J. \& ORTA, B. (1970). Recherche sur l'analyse sérologique des staphylocoques coagulase-négatifs. Etude de deux sérotypes. Annales de microbiologie 119, 193-205.

Pillet, J. \& Orta, B. (1973). Etude sérologique de souches de Staphylococcus aureus d'origine aviaire. Mise en évidence de deux nouveaux sérotypes. Annales de microbiologie 124A, 207-214.

Pitcher, D. G., OWen, R. J., Dyal, P. \& Beck, A. (1987). Synthesis of a biotinylated DNA probe to detect ribosomal RNA cistrons in Providencia stuartii. FEMS Microbiology Letters 48, 283-287.

Saunders, N. A., Harrison, T. G., Kachwalla, N. \& TAYLOR, A. G. (1988). Identification of species of the genus Legionella using a cloned rRNA gene from Legionella pneumophila. Journal of General Microbiology 134, 2363-2374.

SCHLEIFER, K. H., KilpPeR-BÄLZ, R. \& DeVRIESE, L. A. (1984). Staphylococcus arlettae sp. nov., $S$. equorum sp. nov. and $S$. kloosii sp. nov.: three new coagulase-negative, novobiocin-resistant species from animals. Systematic and Applied Microbiology 5 , 501-509.

Southern, E. M. (1975). Detection of specific sequences among DNA fragments separated by gel electrophoresis. Journal of Molecular Biology 98, 503-517.

Stull, T. L., Lipuma, J. J. \& Edling, T. D. (1988). A broad-spectrum probe for molecular epidemiology of bacteria: ribosomal RNA. Journal of Infectious Diseases 157, 280-286.

SUTCLIFFE, J. C. (1978). pBR322 restriction map derived from the DNA sequence: accurate DNA size markers up to 4361 nucleotide pairs long. Nucleic Acids Research 5, 2721-2728.

Yogev, D., Halachmi, D., Kenny, G. E. \& Razin, S. (1988). Distinction of species and strains of mycoplasmas (Mollicutes) by genomic DNA fingerprints with an rRNA gene probe. Journal of Clinical Microbiology 26, 1198-1201. 